\title{
Variations within the compact jet from the black hole candidate GRS 1915+105
}

\section{A. Rushton ${ }^{* \dagger}$}

Jodrell Bank Centre for Astrophysics, School of Physics and Astronomy, University of Manchester, Manchester, M13 9PL

E-mail: Anthony.Rushton [at] manchester.ac.uk

\section{R. Spencer}

Jodrell Bank Centre for Astrophysics, School of Physics and Astronomy, University of Manchester, Manchester, M13 9PL

\section{R. Fender}

School of Physics and Astronomy, University of Southampton, Southampton, SO17 1BJ

Astronomical Institute 'Anton Pannekoek', Univeristy of Amsterdam, Kruislaan 403, 1098 SJ

Amsterdam, the Netherlands

\section{G. Pooley}

The University of Cambridge, Mullard Radio Astronomy Observatory, Cavendish Laboratory, J.

J. Thomson Avenue, Cambridge CB3 OHE

High-resolution observations of the black hole candidate GRS 1915+105 have shown the radio emission to be dominated by a compact steady jet ( $<20$ mas), whilst in the low/hard state (e.g. Dhawan et al. 2000). We have found a close relationship between the X-ray and radio emission from the steady jet using the Ryle Telescope and RXTE-ASM. This was fitted by an empirical relationship of $S_{\mathrm{X}-\text { ray }} \propto S_{\text {radio }}^{\xi}$, with a power-law index of $\xi \sim 1.7$. This suggests either a turn-over of the Gallo et al. (2003) universal XRB relationship, from a radiatively efficient to inefficient accretion disk or direct evidence of a variation in another fundamental parameter, e.g. accretion radius

Science and Technology of Long Baseline Real-Time Interferometry:

The 8th International e-VLBI Workshop, EXPReSO9

June 22 - 262009

Madrid, Spain

\footnotetext{
*Speaker.

${ }^{\dagger}$ www.jb.man.ac.uk/ arushton
} 


\section{Introduction}

It has been observationally established that the formation of astrophysical jets are synonymous with accretion disks in X-ray binaries. Also it has been clearly established that changes in the X-ray spectral state are associated with superluminal knots [1]; however, large scale structures quickly become divorced from the central accretion region as they move away and typically last for many days or weeks. To study the direct coupling between the accretion disk and jet, observations of the compact, 'steady jet' must be selected. As VLBI observations have shown this emission region to be only a few light hours in size [2], changes in the accretion disk may directly change the steady jet.

This paper presents a clear relationship between the accretion disk and the compact jet of one of the most powerful black holes in the Galaxy, GRS 1915+105. As variations in the X-ray emission are associated with changes in the accretion disk, coupling these radio observations to simultaneous $\mathrm{X}$-ray observations can directly relate the inflow-outflow mechanisms around the black hole. For the first time, observations taken over ten years have made it possible to clearly study the repetitive trends between the X-ray and radio. The results presented in this paper select radio and X-ray monitoring observations during the steady jet ('plateau') state. A direct relationship between the inflowing accretion and outflow jet can now be made for GRS 1915+105.

\section{Observations and results}

Observations from the last decade were obtained from instruments monitoring GRS 1915+105 in the radio and X-ray bands. Near daily observations were collected by the Ryle Telescope between May 1995 (MJD 49856) and June 2006 (MJD 53898) at 15 GHz. These data were then compared to 2-12 keV X-ray data taken by the All Sky Monitor on board the RXTE satellite.

Data from each of the two instruments were binned into daily averages, and observations taken on the same day were cross-correlated to form a comprehensive radio-X-ray comparison. Whilst intra-day variability in both X-ray [3] and radio [4] is known to occur for various states, the aim of the work was not to study the details of a particular flare; rather, the goal was to study any longer term correlation (i.e. longer than a few days) between the radio and X-ray in the steady jet state.

Moreover, GRS 1915+105 does not always produce compact self-absorbed emission and largescale, optically thin structure is frequently observed after a state change. Only when a compact jet is present can a direct comparison between the radio and X-ray be made. Therefore, it was important to identify the states that were not contaminated by large scale extended knots [5].

\subsection{Steady jet state}

The clearest correlation between radio and X-ray flux observed in GRS 1915+105 was during the persistent/slightly harder X-ray state (as marked in green in Figure 1). When the X-ray flux is persistently (i.e. more than a few days) between $30-50$ counts/second and has a hardness ratio of HR2 $>1.5$, the source is always associated with strong radio flux. This state was first identified as the 'plateau' state in the radio [4] and state $\mathrm{C}$ with the $\mathrm{X}$-rays [3]. It is also the state where a compact jet is always present $[2,6]$. 


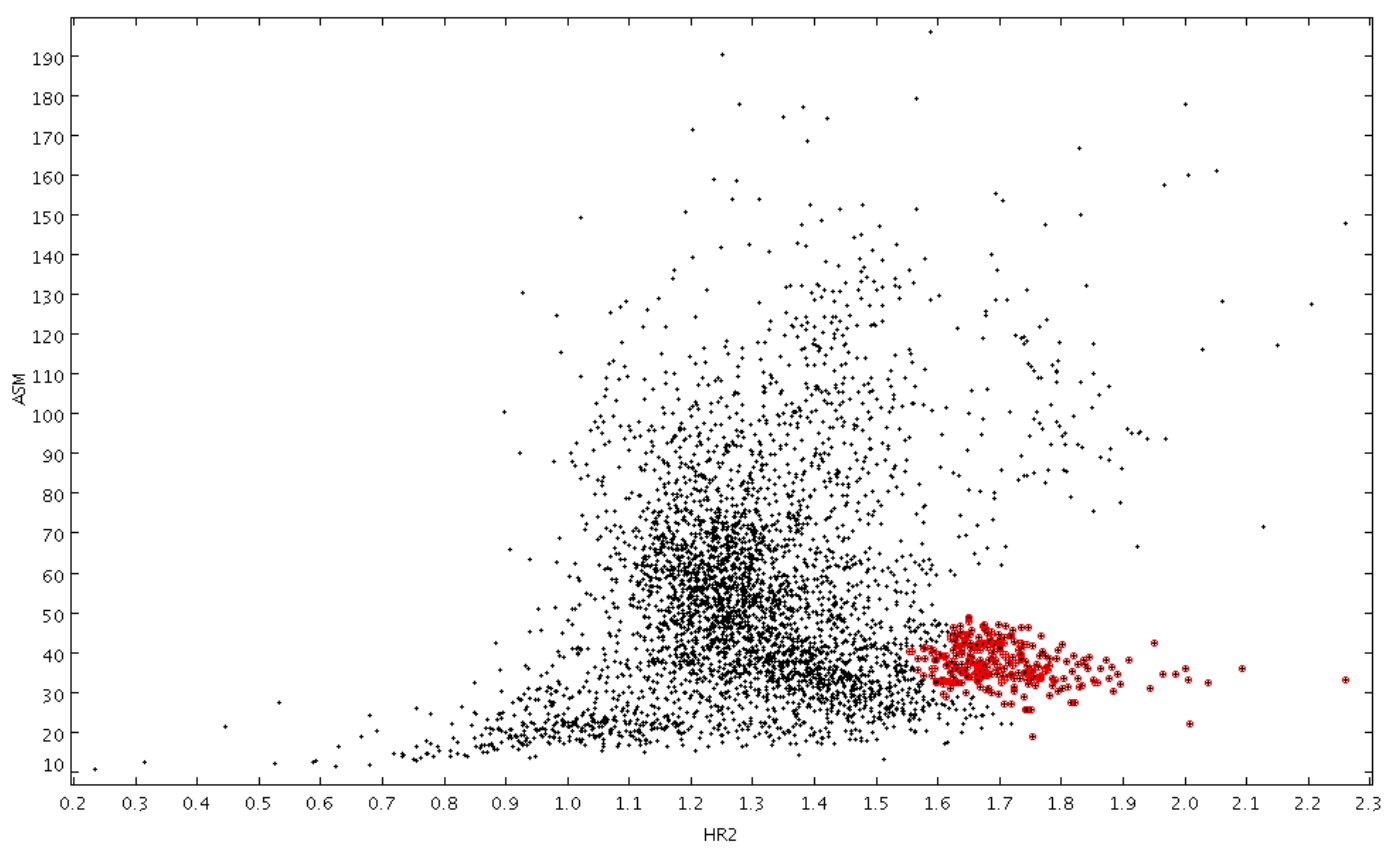

Figure 1: Hardness intensity diagram of GRS 1915+105 between 1996 and 2008, showing the one day average intensity, between $2-12 \mathrm{keV}$, plotted as a function of the hardness radio, HR $2\left(\frac{5-12 \mathrm{keV}}{2-5 \mathrm{keV}}\right)$. The dots circles in red represent persistent radio emission associated with the $\mathrm{X}$-rays, also known as the class $\chi \mathrm{X}$-ray state in the [3] classification or the 'plateau' state in [4].

The X-ray and radio lightcurves of GRS 1915+105 over the ten year period between 1996 and 2006 are shown in Figure 2. Marked in red are the points selected in the persistent/slightly harder $\mathrm{X}$-ray state, that are also identified as green on Figure 1 (i.e. the radio plateau/steady jet state). It is clear that the long flaring periods of radio activity are coincident with this X-ray state. Furthermore, both the X-ray and radio emission appear to decay during each flaring period. Table 1 lists eight periods of sustained flaring activity (ranging from 4 to 63 days) identified between January 1996 (MJD 50110) and May 2006 (MJD 53883). It thus appears that state $C$ or the 'plateau' state occurs approximately every 1.3 years.

\section{Disscussion and Conclusions}

A power-law function of $S_{\text {radio }} \propto S_{\mathrm{X} \text {-ray }}^{\xi}$ has been fitted to the data, which gave a best fit of $\xi \sim 1.72 \pm 0.13$ (see Figure 3). This is much steeper than the previous correlations that have shown $\xi \sim 0.7$ for XRBs in the low-hard state [7]. Previous models have then suggested that the bolometric luminosity is dominated by an advection flow and thus the observed X-ray emission is $L_{\mathrm{X}-\text { ray }} \propto \dot{m}^{2}$ [8]. As the radio emission relates to the accretion rate as $L_{\text {radio }} \propto \dot{m}^{12 / 17}$, the radio emission couples to the X-ray emission as $L_{\mathrm{rad}} \propto L_{\mathrm{X}}^{0.7}$, which is a radiatively inefficient accretion. However, the fit has a much steeper slope that can be fitted without assuming an advection flow. If the X-ray emission coupled linearly, $L_{\mathrm{X} \text {-ray }} \propto \dot{m}$, with the accretion rate [9] then $L_{\mathrm{rad}} \propto L_{\mathrm{X}}^{1.4}$, which is a radiatively efficient accretion and gives a much closer fit to the observed relationship. 


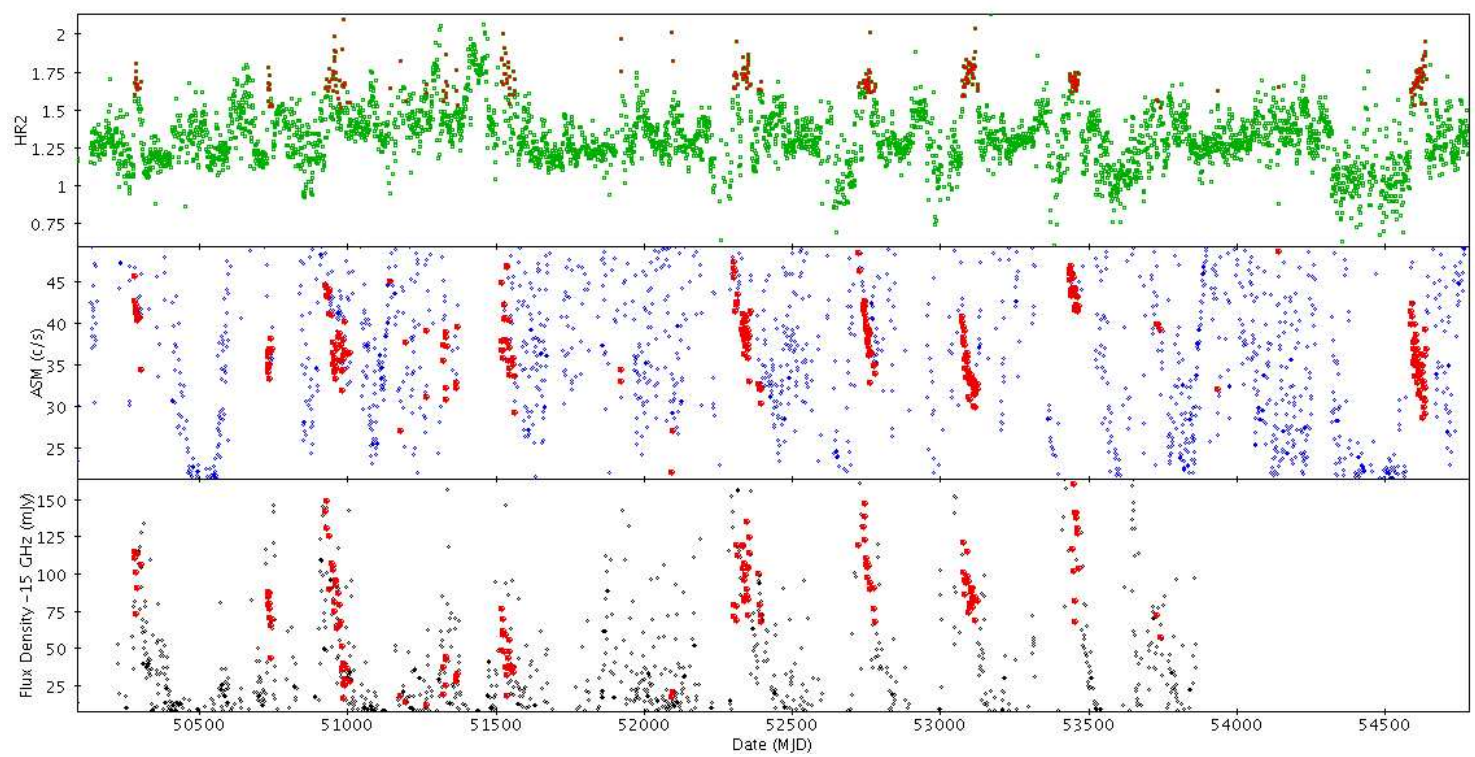

Figure 2: X-ray and radio lightcurves of GRS 1915+105 over a ten year period with the red marks representing observations taken in the State $\mathrm{C}$ or 'plateau' state (i.e. ASM counts of $30-50$ per second and HR2 > 1.5); the black points are $15 \mathrm{GHz}$ radio observations taken by the Ryle Telescope, the blue and green points are $2-12 \mathrm{keV}$ X-ray and HR2 hardness ratio $\left(\frac{5-12 \mathrm{keV}}{2-5 \mathrm{keV}}\right)$ observations taken by the RXTE-ASM. The top graph shows all observations combined and the bottom graph shows the same data with the intensity axis scaled to show the selected observations.

\begin{tabular}{|ll|ccc|}
\hline $\begin{array}{l}\text { Start date } \\
(\mathrm{MJD})\end{array}$ & $\begin{array}{l}\text { End date } \\
(\mathrm{MJD})\end{array}$ & $\begin{array}{c}\text { Change in X-rays }^{\dagger} \\
(\text { counts/second })\end{array}$ & $\begin{array}{c}\text { Change in radio } \\
(\mathrm{mJy})\end{array}$ & $\begin{array}{c}\text { Duration } \\
(\text { Days })\end{array}$ \\
\hline 50286 & 50297 & $42 \rightarrow 40$ & $110 \rightarrow 100$ & 11 \\
50732 & 50736 & $36 \rightarrow 33$ & $94 \rightarrow 87$ & 4 \\
50926 & 50989 & $45 \rightarrow 34$ & $154 \rightarrow 31$ & 63 \\
51522 & 51546 & $39 \rightarrow 33$ & $78 \rightarrow 50$ & 24 \\
52315 & 52354 & $43 \rightarrow 36$ & $122 \rightarrow 73$ & 41 \\
52729 & 52783 & $46 \rightarrow 33$ & $163 \rightarrow 68$ & 54 \\
53077 & 53116 & $39 \rightarrow 31$ & $122 \rightarrow 70$ & 39 \\
53416 & 53466 & $52 \rightarrow 42$ & $197 \rightarrow 112$ & 50 \\
\hline
\end{tabular}

Table 1: List of the low/hard states (State C) states of GRS 1915+105 between January 1996 (MJD 50110) and May 2006 (MJD 53883). The X-ray observations were measured as one day averages of the RXTE-ASM observations between 2-12 keV. Radio observations were taken at $15 \mathrm{GHz}$ with the Ryle Telescope (RT). $\dagger$ RXTE-ASM errors were typically less than 1 count/second. $\ddagger$ RT errors are estimated at approximately $\pm 6 \mathrm{mJy}$ 


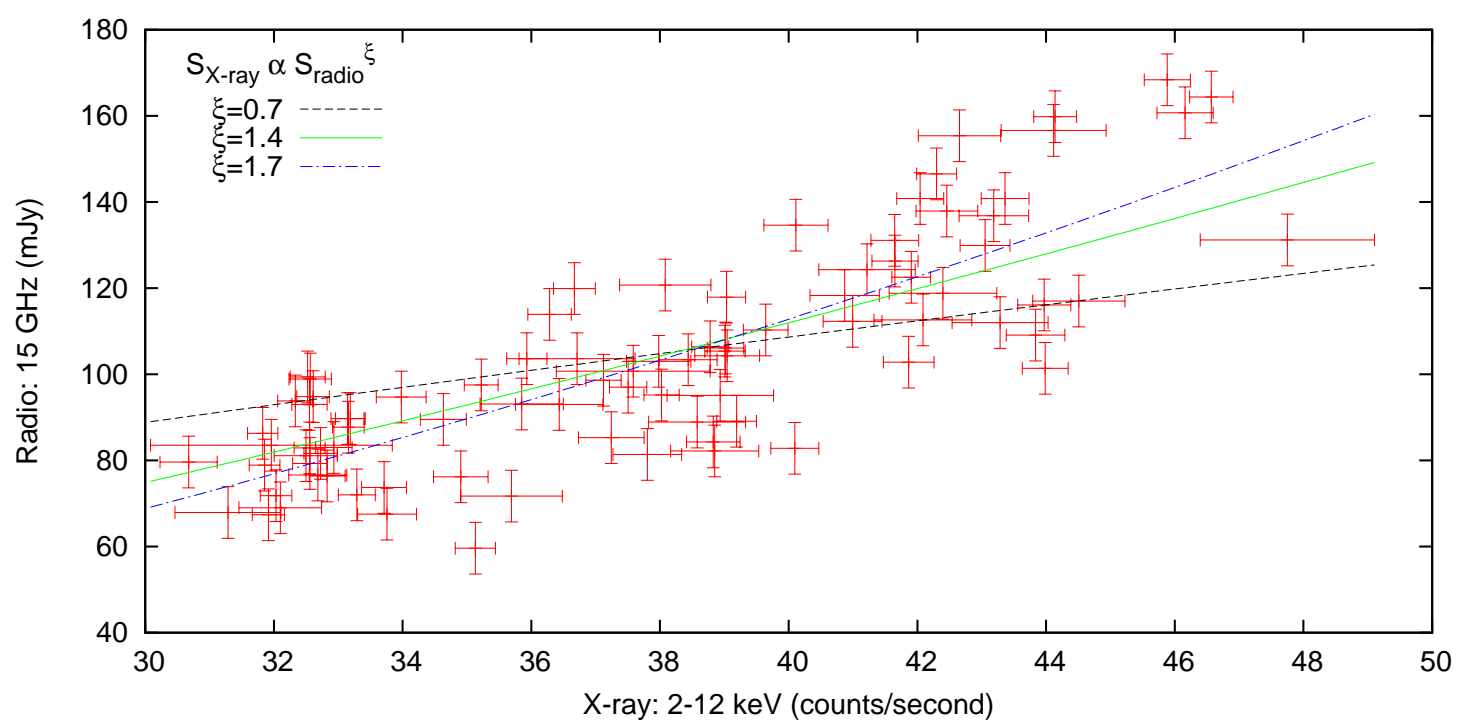

Figure 3: X-ray/Radio correlation in the low-hard state of GRS 1915+1915 (i.e. steady jet state). The dashdotted-blue line represents a 'best-fit' of $\xi \sim 1.72 \pm 0.13$, the solid-green line represent a model of $\xi \sim 1.4$ and the dashed-black line represents a model of $\xi \sim 0.7$, where $S_{\mathrm{X}-\text { ray }} \propto S_{\text {radio }}^{\xi}$.

It has been suggested that the collapse of the inner region $\left(r_{\text {in }}\right)$ of a thin disk occurs during a transition to the harder X-ray state (state C) [10]. However, the X-ray emission is a factor of $\sim 100$ brighter in GRS 1915+105 (and relatively softer) than most XRBs observed in the low/hard state. Furthermore, XMM-Newton observations [11] suggest the presence of an optically thick reflector, that provides evidence of the presence of a thin disk in the X-ray spectrum. It is therefore suggested that the dominant mode of accretion, observed in X-ray emission, is above $r_{\text {in }}$ and is emitted by a radiatively efficient thin disk, where $L_{\mathrm{X}} \propto \dot{m}_{\mathrm{in}}$, rather an advection dominated flow. The emission mechanism could be a non-thermal process, such as inverse Compton scattering, where the thin disk can cool via the scattering by cold electrons in a corona. Most of the heat would therefore be lost before reaching $r_{\text {in }}$.

There could be a limited rate at which the dominant mode of accretion changes from radiatively inefficient to radiatively efficient. For XRBs this is about $\dot{m} \sim 10^{18.5} \mathrm{~g} / \mathrm{s}$. For large black holes (such as AGN) this value scales with mass and this would mean advection dominated flows are not sustainable at a very high fraction of the Eddington accretion rate of about $\dot{m}>0.1 \dot{m}_{\text {edd }}$.

Alternatively, at the very high mass accretion rates, there could be a turn-over in the dominance of the bolometric luminosity between the accretion disk and the jet. The X-ray luminosity could become dominated by synchrotron or self-comptonization, producing the non-thermal X-ray emission. The bolometric luminosity would simply become proportional to the out-flow rate $\dot{m}_{\text {jet }}$ and thus $\propto Q_{\text {jet }}$, the jet power .

This work has shown, for the first time, a direct relationship between the X-rays and radio in the steady jet state of GRS 1915+105. Previous attempts have failed to show this relationship [12], as they have included X-ray/radio comparisons that include extended knots and or X-ray emission 
in other states. This is also the first time that a radiatively efficient accretion process has been observed in a black hole XRB, whereas previous radio-X-ray correlations have only observed radiatively inefficient accretion [7]. The difference between different XRBs is likely to be due to the rate of accretion. GRS 1915+105 is known to be in a constant 'soft'-like state as a large accretion rate is constantly present; however, it remains unclear if the X-ray emission, in the steady jet state, is produced from either the thin disk, an advection dominated flow or the compact jet. For other XRBs, the bright sources are likely to form only a transient accretion disk and GRS 1915+105-type accretion may only occur in systems with higher accretion rates, like AGN.

\section{Acknowledgements}

AR acknowledges support from an STFC studentship during this research and part of this work was also supported by the EXPReS project. Thanks are also given to Tom Maccarone and Elena Gallo for useful discussion throughout this work. EXPReS is an Integrated Infrastructure Initiative (I3), funded under the European Commission's Sixth Framework Programme (FP6), contract number 026642. The Ryle Telescope is operated by the University of Cambridge and supported by STFC. The X-ray data was provided by the ASM/RXTE teams at MIT and at the RXTE SOF and GOF at NASA's GSFC.

\section{References}

[1] Fender, R. P., Garrington, S. T., McKay, D. J., Muxlow, T. W. B., Pooley, G. G., Spencer, R. E., Stirling, A. M., \& Waltman, E. B. 1999, MNRAS, 304, 865

[2] Dhawan, V., Mirabel, I. F., \& Rodríguez, L. F. 2000, ApJ, 543, 373

[3] Belloni, T., Klein-Wolt, M., Méndez, M., van der Klis, M., \& van Paradijs, J. 2000, A\&A, 355, 271

[4] Pooley, G. G., \& Fender, R. P. 1997, MNRAS, 292, 925

[5] Mirabel, I. F., \& Rodríguez, L. F. 1994, Nat, 371, 46

[6] Fuchs, Y., et al. 2003, A\&A, 409, L35

[7] Gallo, E., Fender, R. P., \& Pooley, G. G. 2003, MNRAS, 344, 60

[8] Rees, M. J., Begelman, M. C., Blandford, R. D., \& Phinney, E. S. 1982, Nat, 295, 17

[9] Shakura, N. I., \& Sunyaev, R. A. 1973, A\&A, 24, 337

[10] Migliari, S., \& Belloni, T. 2003, A\&A, 404, 283

[11] Martocchia, A., Matt, G., Belloni, T., Feroci, M., Karas, V., \& Ponti, G. 2006, A\&A, 448, 677

[12] Muno, M. P., Remillard, R. A., Morgan, E. H., Waltman, E. B., Dhawan, V., Hjellming, R. M., \& Pooley, G. 2001, ApJ, 556, 515 